\title{
ON THE REGULARITY OF THE STATE CONSTRAINED MINIMAL TIME FUNCTION
}

\author{
BY
}

\section{A.I. LAZU}

\begin{abstract}
In this paper we provide a sufficient condition for the state constrained minimal time function to be proto-Lipschitz around the target.

Mathematics Subject Classification 2000: 49N60, 93B05.

Key words: minimal time function, state constrained, proto-Lipschitz property.
\end{abstract}

\section{Introduction}

Let $F$ be a multifunction mapping $\mathbb{R}^{p}$ to subsets of $\mathbb{R}^{p}, K$ a nonempty subset of $\mathbb{R}^{p}$ and $x \in K$. Let us consider the following state constrained differential inclusion

$$
\left\{\begin{array}{l}
y^{\prime}(t) \in F(y(t)) \\
y(0)=x \\
y(t) \in K, \quad \text { for all } t \geq 0 .
\end{array}\right.
$$

A solution of (1.1) on $[0, T]$ is an absolutely continuous function $y:[0, T] \rightarrow$ $K$, with the initial value $y(0)=x$, that satisfies $y^{\prime}(t) \in F(y(t))$ a.e. $t \in$ $[0, T]$. We refer to such an $y$ as a $K$-trajectory (of $F$ ) that originates from $x$.

The $K$-constrained minimal time problem we consider here is that of steering an initial point $x \in K$ to a given target set $\Sigma$ along a $K$-trajectory of $F$ in minimal time. In this paper we consider the case when the target $\Sigma$ is a compact subset of $K$.

The minimal time value is denoted by $T_{K}(x)$, which could be $+\infty$ if no trajectory in $K$ from $x$ can reach $\Sigma$. 
When $K=\mathbb{R}^{p}, T_{K}(\cdot)$ coincides with the well known (unconstrained) minimal time function associated with the target $\Sigma$, denoted by $T(\cdot)$.

The regularity properties of the minimal time function, giving important information on the system, have been the object of an extensive literature. The Lipschitz continuity of the unconstrained minimal time function $T(\cdot)$ was first studied in [12] for $\Sigma=\{0\}$. In that paper, Petrov introduced a necessary and sufficient condition, called Petrov condition, under which $T(\cdot)$ is Lipschitz continuous in a neighborhood of the origin. That result was extended later to more general target sets (see, e.g. [13, 2]). In [14], Veliov obtained a necessary and sufficient condition for the local Lipschitz continuity of $T(\cdot)$, when the multifunction $F$ is nonautonomous and depends measurably on the time. In [15] Wolenski and Zhuang showed that the Lipschitz continuity of $T(\cdot)$ near $\Sigma$ is equivalent to the boundedness of the proximal subgradient of $T(\cdot)$ for points in $\Sigma$. See also [4], [5], [6], [7] for some regularity results of the minimal time function associated with semilinear control systems in Banach spaces.

The state constrained case was considered in [11]. In that paper, Nour and Stern generalized the results obtained for the unconstrained minimal time function in [15]. They gave necessary and sufficient conditions for the proto-Lipschitzness of $T_{K}(\cdot)$, imposing the admissibility of the target set $\Sigma$ for $K$ and conditions involving points near $\Sigma$ which are exterior to $K$.

In our paper we provide a sufficient condition for the minimal time function $T_{K}(\cdot)$ to be locally proto-Lipschitz, completely different from the ones in [11], without requiring hypothesis of admissibility or conditions over points exterior to $K$.

\section{Preliminaries}

Throughout this paper we denote by $\|\cdot\|,\langle\cdot, \cdot\rangle$, respectively, the Euclidean norm and scalar product in $\mathbb{R}^{p}$. For any subset $S \subset \mathbb{R}^{p}$, bdryS stands for its boundary and $S^{c}$ for its complement. We denote by $\pi_{S}(x)$ the set of projections of $x \in \mathbb{R}^{p}$ in $S$ and by $d_{S}(x)$ the Euclidean distance from $x$ to $S$. The open unit ball is denoted by $B$.

A vector $\eta \in \mathbb{R}^{p}$ is tangent to $S$ at a point $\xi \in S$ if

$$
\liminf _{h \downarrow 0} \frac{1}{h} d_{S}(\xi+h \eta)=0 .
$$

We denote by $\mathcal{T}_{S}(\xi)$ the set of all vectors which are tangent to the set $S$ at the point $\xi$. The following characterization is useful below. 
Namely, $\eta \in \mathcal{T}_{S}(\xi)$ if and only if there exist two sequences $\left(h_{n}\right)_{n}$ in $\mathbb{R}_{+}$ with $h_{n} \downarrow 0$ and $\left(p_{n}\right)_{n}$ in $\mathbb{R}^{p}$ with $\lim _{n} p_{n}=0$ such that $\xi+h_{n}\left(\eta+p_{n}\right) \in S$ for each $n \in \mathbb{N}$.

Let $\mathcal{F}: S \rightsquigarrow \mathbb{R}^{p}$ be a given multifunction and consider the differential inclusion

$$
w^{\prime}(t) \in \mathcal{F}(w(t))
$$

The set $S$ is viable with respect to $\mathcal{F}$ if for each $\xi \in S$ there exists $T>0$ such that (2.2) has at least one solution $w:[0, T] \rightarrow S$ with $w(0)=\xi$.

We present now a viability result useful in our study (see $[8$, Theorem 6.2.4]). See also [1], [10].

Theorem 2.1. Let $S$ be a nonempty and locally closed subset in $\mathbb{R}^{p}$ and let $\mathcal{F}: S \rightsquigarrow \mathbb{R}^{p}$ be an upper semicontinuous multifunction with nonempty, compact and convex values. A necessary and sufficient condition in order that $S$ be viable with respect to $\mathcal{F}$ is that

$$
\mathcal{F}(\xi) \cap \mathcal{T}_{S}(\xi) \neq \emptyset
$$

for each $\xi \in S$.

We end this section with the statement of the Brezis-Browder ordering principle following the presentation given in [8, Theorem 2.1.1]. See [3] for the original result.

Theorem 2.2. Let $\mathcal{S}$ be a nonempty set, $\preceq \subseteq \mathcal{S} \times \mathcal{S}$ a preorder on $\mathcal{S}$ and let $\mathcal{N}: \mathcal{S} \rightarrow \mathbb{R} \cup\{+\infty\}$ be a function. Suppose that:

(i) each increasing sequence in $\mathcal{S}$ is bounded from above;

(ii) the function $\mathcal{N}$ is increasing.

Then, for each $\xi_{0} \in \mathcal{S}$, there exists an $\mathcal{N}$-maximal element $\bar{\xi} \in \mathcal{S}$ satisfying $\xi_{0} \preceq \bar{\xi}$.

Recall that $\bar{\xi} \in \mathcal{S}$ is $\mathcal{N}$-maximal if $\mathcal{N}(\xi)=\mathcal{N}(\bar{\xi})$ for every $\xi \in \mathcal{S}$ with $\bar{\xi} \preceq \xi$. We mention that in [3] the function $\mathcal{N}$ is considered to be bounded above. The extension to the general case above was first considered in [9]. 


\section{Regularity results}

Let $K \subset \mathbb{R}^{p}$ be a closed nonempty set and let $\Sigma \subset K$ be a compact subset. The $K$-constrained minimal time function $T_{K}: K \rightarrow[0,+\infty]$ is defined by

$T_{K}(x)=\inf \{T \geq 0 ; \exists y(\cdot)$ a $K$-trajectory of $F$ with $y(0)=x, y(T) \in \Sigma\}$. If no $K$-trajectory from $x$ can reach $\Sigma$ then $T_{K}(x)=+\infty$.

The case when $\Sigma=K$ is trivial as $T_{K}(x)=0$ for any $x \in K$. Throughout the paper we shall consider the set $K \backslash \Sigma$ to be nonempty.

Following [11], we define the proto-Lipschitz property.

Definition 3.1. We say that $T_{K}(\cdot)$ is proto-Lipschitz on a neighborhood of $\Sigma$ in $K$ if there exist $\rho>0$ and $M>0$ such that

$$
T_{K}(x) \leq M d_{\Sigma}(x)
$$

for all $x \in(\Sigma+\rho B) \cap K$.

We state the main result of the paper, which provides a sufficient condition for the proto-Lipschitzness of the $K$-constrained minimal time function $T_{K}(\cdot)$. We shall use the convention that $\inf \emptyset=\infty$.

Theorem 3.2. Let $F: \mathbb{R}^{p} \rightsquigarrow \mathbb{R}^{p}$ be an upper semi-continuous multifunction with nonempty compact and convex values. Suppose that there exist $r>0, c>0$ and $\gamma>0$ such that

$$
\inf _{s \in \pi_{\Sigma}(x)} \inf _{u \in F(x) \cap \mathcal{T}_{K}(x)}\langle x-s, u\rangle \leq c d_{\Sigma}^{2}(x)-\gamma d_{\Sigma}(x),
$$

for all $x \in K \cap(\Sigma+r B)$. Then the $K$-constrained minimal time function $T_{K}(\cdot)$ is locally proto-Lipschitz. More precisely, for any $0<\rho<\min \left\{r, \frac{\gamma}{c}\right\}$ we have that

$$
T_{K}(x) \leq \frac{d_{\Sigma}(x)}{\gamma-c \rho}
$$

for all $x \in K \cap(\Sigma+\rho B)$.

Remark 3.3. Let us note that the hypothesis that there exist $r>0$, $c>0$ and $\gamma>0$ such that (3.4) holds is, in fact, equivalent to the apparently stronger one: there exist $r^{\prime}>0$ and $\gamma^{\prime}>0$ such that

$$
\inf _{s \in \pi_{\Sigma}(x)} \inf _{u \in F(x) \cap \mathcal{T}_{K}(x)}\langle x-s, u\rangle \leq-\gamma^{\prime} d_{\Sigma}(x),
$$


for all $x \in K \cap\left(\Sigma+r^{\prime} B\right)$. In this setting, the estimate (3.5) takes the form

$$
T_{K}(x) \leq \frac{d_{\Sigma}(x)}{\gamma^{\prime}}
$$

for all $x \in K \cap\left(\Sigma+r^{\prime} B\right)$.

The following result gives a priori estimates for the initial value problem $(1.1)$.

Theorem 3.4. Let $F$ be an upper semi-continuous multifunction with nonempty compact and convex values. Suppose that there exist $r>0, c>0$ and $\gamma>0$ such that (3.4) holds for all $x \in K \cap(\Sigma+r B)$. Then, for each $\rho \leq r$ and $x \in K \cap(\Sigma+\rho B) \backslash \Sigma$ there exists $y:[0, \tau) \rightarrow K \cap(\Sigma+\rho B) \backslash \Sigma a$ noncontinuable solution of (1.1) which satisfies the inequality

$$
d_{\Sigma}(y(t)) \leq e^{c t}\left(d_{\Sigma}(x)-\frac{\gamma}{c}\right)+\frac{\gamma}{c}
$$

for all $t \in[0, \tau)$.

Proof. Step 1 First, we prove that, for any $\rho \leq r$ and $x \in K \cap$ $(\Sigma+\rho B) \backslash \Sigma$ there exist $T>0$ and $y:[0, T] \rightarrow K \cap(\Sigma+\rho B) \backslash \Sigma$ solution of (1.1) such that

$$
d_{\Sigma}(y(t)) \leq d_{\Sigma}(x)+c \int_{0}^{T} d_{\Sigma}(y(s)) d s-\gamma t
$$

for all $t \in[0, T]$.

Let $\rho \leq r, \widetilde{K}=K \cap(\Sigma+\rho B)$ and consider the set

$$
\mathcal{K}=\left\{(x, \lambda) ; x \in \widetilde{K} \backslash \Sigma, d_{\Sigma}(x) \leq \lambda\right\}
$$

It is easy to see that $\mathcal{K}$ is a nonempty and locally closed set. We define the multifunction $\mathcal{F}: \mathcal{K} \rightsquigarrow \mathbb{R}^{p+1}$ by

$$
\mathcal{F}(y, z)=F(y) \times\left\{c d_{\Sigma}(y)-\gamma\right\},
$$

for all $(y, z) \in \mathcal{K}$ and we consider the problem

$$
\left\{\begin{array}{l}
w^{\prime}(t) \in \mathcal{F}(w(t)) \\
w(0)=\left(x, d_{\Sigma}(x)\right) .
\end{array}\right.
$$


We shall prove that $\mathcal{K}$ is viable with respect to $\mathcal{F}$. To this end we shall apply Theorem 2.1. So, we have to prove a condition of type (2.3) .

Let $(x, \lambda) \in \mathcal{K}$. By $(3.4)$, there exist $s \in \pi_{\Sigma}(x)$ and $u \in F(x) \cap \mathcal{T}_{K}(x)$ such that

$$
\langle x-s, u\rangle \leq c d_{\Sigma}^{2}(x)-\gamma d_{\Sigma}(x),
$$

which is equivalent to

$$
\lim _{t \downarrow 0} \frac{\|x-s+t u\|-d_{\Sigma}(x)}{t} \leq c d_{\Sigma}(x)-\gamma .
$$

As $u \in \mathcal{T}_{K}(x)$, there exist two sequences $h_{n} \downarrow 0$ and $p_{n} \rightarrow 0$ in $\mathbb{R}^{p}$ such that $x+h_{n}\left(u+p_{n}\right) \in K$. We get that

$$
\lim _{n \rightarrow \infty} \frac{d_{\Sigma}\left(x+h_{n} u\right)-d_{\Sigma}(x)}{h_{n}} \leq \lim _{n \rightarrow \infty} \frac{\left\|x+h_{n} u-s\right\|-d_{\Sigma}(x)}{h_{n}} \leq c d_{\Sigma}(x)-\gamma .
$$

Hence, there exist two sequences $r_{n} \downarrow 0$ and $t_{n} \downarrow 0$ (a subsequence of $h_{n}$ ) such that

$$
\frac{d_{\Sigma}\left(x+t_{n} u\right)-d_{\Sigma}(x)}{t_{n}} \leq c d_{\Sigma}(x)-\gamma+r_{n}
$$

for any $n=1,2, \ldots$ Therefore, we obtain that

$$
\begin{aligned}
d_{\Sigma}\left(x+t_{n} u+t_{n} p_{n}\right) & \leq d_{\Sigma}\left(x+t_{n} u\right)+t_{n}\left\|p_{n}\right\| \\
& \leq d_{\Sigma}(x)+t_{n}\left(c d_{\Sigma}(x)-\gamma\right)+t_{n} q_{n} \\
& \leq \lambda+t_{n}\left(c d_{\Sigma}(x)-\gamma\right)+t_{n} q_{n},
\end{aligned}
$$

for any $n=1,2, \ldots$ where $q_{n}:=r_{n}+\left\|p_{n}\right\| \rightarrow 0$. Also, we have that $x+t_{n} u+$ $t_{n} p_{n} \in \widetilde{K}$, for $n$ sufficiently large. So, we conclude that $\left(u, c d_{\Sigma}(x)-\gamma\right) \in$ $\mathcal{T}_{\mathcal{K}}(x, \lambda)$, that is $\mathcal{F}(x, \lambda) \cap \mathcal{T}_{\mathcal{K}}(x, \lambda) \neq \emptyset$ and, by Theorem 2.1, we get the viability of $\mathcal{K}$ with respect to $\mathcal{F}$. Then, there exist $T>0$ and $w:[0, T] \rightarrow \mathcal{K}$ solution of problem (3.8), i.e., there exists $y:[0, T] \rightarrow \widetilde{K} \backslash \Sigma$ solution of (1.1) such that

$$
d_{\Sigma}(y(t)) \leq d_{\Sigma}(x)+c \int_{0}^{t} d_{\Sigma}(y(s)) d s-\gamma t
$$

for all $t \in[0, T]$. Moreover, by Gronwall inequality we obtain that (3.6) holds for all $t \in[0, T]$.

Step 2 We prove the conclusion of the theorem. To this aim, we shall make use of Brezis-Browder ordering principle, Theorem 2.2. 
Let $x \in \widetilde{K} \backslash \Sigma$. Let $\mathcal{S}$ be the set of all the solutions $y_{a}(\cdot), a>0$, of (1.1), $y_{a}:[0, a) \rightarrow \widetilde{K} \backslash \Sigma$, satisfying (3.7) for all $t \in[0, a)$. This set is clearly nonempty, as we have already proved. We introduce a preorder on $\mathcal{S}$ as follows. We say that $y_{a} \preceq y_{b}(a, b>0)$ if $a \leq b$ and $y_{a}(t)=y_{b}(t)$, for all $t \in[0, a)$. Also, we introduce the increasing function $\mathcal{N}: \mathcal{S} \rightarrow \mathbb{R} \cup\{+\infty\}$ defined by $\mathcal{N}\left(y_{a}\right)=a$, for any $y_{a} \in \mathcal{S}$. We have to prove that each increasing sequence in $\mathcal{S}$ is bounded from above. Indeed, let $\left(y_{n}\right)_{n}$ be an increasing sequence in $\mathcal{S}, y_{n}:\left[0, a_{n}\right) \rightarrow \widetilde{K} \backslash \Sigma, n=1,2, \ldots$ and define $\widetilde{y}:\left[0, \sup _{n} a_{n}\right) \rightarrow$ $\widetilde{K} \backslash \Sigma$, by $\widetilde{y}(t)=y_{n}(t)$, for any $t \in\left[0, a_{n}\right)$. Then $\widetilde{y}(\cdot)$ is well defined, verifies (3.7) for all $t \in\left[0, \sup _{n} a_{n}\right)$, so $\widetilde{y} \in \mathcal{S}$, and $\widetilde{y}$ is an upper bound for $\left(y_{n}\right)_{n}$. Consequently the set $\mathcal{S}$ endowed with the preorder $\preceq$ and the function $\mathcal{N}$ satisfy the hypotheses of Brezis-Browder Ordering Principle. Accordingly, there exists an $\mathcal{N}$-maximal element $\bar{y} \in \mathcal{S}, \bar{y}:[0, \bar{a}) \rightarrow \widetilde{K} \backslash \Sigma$, such that, if $\bar{y} \preceq \widetilde{y}, \widetilde{y} \in \mathcal{S}$, then $\mathcal{N}(\widetilde{y})=\mathcal{N}(\bar{y})$.

Now, we prove that the solution $\bar{y}:[0, \bar{a}) \rightarrow \widetilde{K} \backslash \Sigma$ of $(1.1)$ is noncontinuable. Assume by contradiction that $\bar{y}(\cdot)$ is continuable. Then, there exist $\sigma>\bar{a}$ and $\widetilde{y}:[0, \sigma) \rightarrow \widetilde{K} \backslash \Sigma$ such that $\widetilde{y}(t)=\bar{y}(t)$, for all $t \in[0, \bar{a})$. Then, we have that $d_{\Sigma}(\widetilde{y}(\bar{a})) \leq e^{c \bar{a}}\left(d_{\Sigma}(x)-\frac{\gamma}{c}\right)+\frac{\gamma}{c}$.

If $d_{\Sigma}(\widetilde{y}(\bar{a}))<e^{c \bar{a}}\left(d_{\Sigma}(x)-\frac{\gamma}{c}\right)+\frac{\gamma}{c}$, then there exists $b>\bar{a}$ such that $d_{\Sigma}(\widetilde{y}(t))<e^{c t}\left(d_{\Sigma}(x)-\frac{\gamma}{c}\right)+\frac{\gamma}{c}$, for any $t \in[\bar{a}, b)$, so we obtain a contradiction. Suppose now that

$$
d_{\Sigma}(\widetilde{y}(\bar{a}))=e^{c \bar{a}}\left(d_{\Sigma}(x)-\frac{\gamma}{c}\right)+\frac{\gamma}{c} .
$$

As $\widetilde{y}(\bar{a}) \in \widetilde{K} \backslash \Sigma$, by the first part of the proof and (3.10), there exist $b>\bar{a}$ and a solution $\widehat{y}:[\bar{a}, b) \rightarrow \widetilde{K} \backslash \Sigma$ of $(1.1)$ with $d_{\Sigma}(\widehat{y}(t)) \leq e^{c(t-\bar{a})}\left(d_{\Sigma}(\widetilde{y}(\bar{a}))-\right.$ $\left.\frac{\gamma}{c}\right)+\frac{\gamma}{c}=e^{c t}\left(d_{\Sigma}(x)-\frac{\gamma}{c}\right)+\frac{\gamma}{c}$, for $t \in[\bar{a}, b)$, which also leads to a contradiction.

Corollary 3.5. Assume the hypotheses of Theorem 3.4. Then, for each $\rho \leq \min \left\{r, \frac{\gamma}{c}\right\}$ and $x \in K \cap(\Sigma+\rho B) \backslash \Sigma$ there exists a $K$-trajectory of (1.1) that reaches the target $\Sigma$ in some time

$$
\tau \leq \frac{1}{c} \ln \frac{\frac{\gamma}{c}}{\frac{\gamma}{c}-d_{\Sigma}(x)} .
$$

Proof. Let $\rho \leq \min \left\{r, \frac{\gamma}{c}\right\}$ and $x \in \widetilde{K} \backslash \Sigma$, where $\widetilde{K}=K \cap(\Sigma+\rho B)$. By Theorem 3.4 there exists $y:[0, \tau) \rightarrow \widetilde{K} \backslash \Sigma$ a noncontinuable solution of (1.1) 
which satisfies the inequality (3.6) for all $t \in[0, \tau)$. If $\tau=+\infty$, we obtain that $d_{\Sigma}(y(t)) \neq 0$ for all $t>0$ which contradicts inequality (3.6). Hence $\tau<+\infty$. Also, from (3.6), we obtain that $y(\cdot)$ is bounded on $[0, \tau)$. As $F$ maps bounded sets into bounded sets, we have that $F(y(\cdot))$ is bounded on $[0, \tau)$. Then there exists $\lim _{t \uparrow \tau} y(t)$ and belongs to $K$. Passing to the limit for $t \uparrow \tau$ in (3.6) we obtain that $d_{\Sigma}\left(\lim _{t \uparrow \tau} y(t)\right) \leq e^{c \tau}\left(d_{\Sigma}(x)-\frac{\gamma}{c}\right)+\frac{\gamma}{c}<\rho$ because $d_{\Sigma}(x)<\rho \leq \frac{\gamma}{c}$, so $\lim _{t \uparrow \tau} y(t) \in \widetilde{K}$. If $d_{\Sigma}\left(\lim _{t \uparrow \tau} y(t)\right) \neq 0$, then, by Theorem 3.4, we obtain that $y$ can be continued to the right of $\tau$, but this is false. In conclusion, $\lim _{t \uparrow \tau} y(t) \in \Sigma$. The estimate (3.11) follows easily from (3.6) .

We continue with the proof of Theorem 3.2.

Proof. From the previous Corollary we obtain that, for each $x \in K \cap$ $(\Sigma+r B)$ with $0<d_{\Sigma}(x)<\frac{\gamma}{c}$,

$$
T_{K}(x) \leq \frac{1}{c} \ln \frac{\frac{\gamma}{c}}{\frac{\gamma}{c}-d_{\Sigma}(x)} .
$$

Moreover,

$$
T_{K}(x) \leq \frac{1}{c} \ln \left(1+\frac{d_{\Sigma}(x)}{\frac{\gamma}{c}-d_{\Sigma}(x)}\right) \leq \frac{d_{\Sigma}(x)}{\gamma-c d_{\Sigma}(x)} .
$$

In conclusion, for any $0<\rho<\min \left\{r, \frac{\gamma}{c}\right\}$ we have

$$
T_{K}(x) \leq \frac{d_{\Sigma}(x)}{\gamma-c \rho}
$$

for all $x \in(\Sigma+\rho B) \cap K$.

\section{Examples}

In [11] there are given necessary and sufficient conditions for the protoLipschitzness of $T_{K}(\cdot)$, under certain hypotheses regarding points near the target $\Sigma$ which are exterior to $K$. Also, the admissibility of $\Sigma$ for $K$ is required. The hypotheses of Theorem 3.2 do not involve points exterior to $K$ or the admissibility of $\Sigma$ for $K$. There are systems that do not satisfy the conditions imposed in [11], but satisfy our hypotheses. The following examples illustrate this statement. 
Example 4.1. Consider $K=\{(x, y) ; y \geq 0\}, \Sigma=\{(0,0)\}$ and $F(x, y)$ $=B \cap\{(x, y) ; y \leq 0\}$ for all $(x, y) \in \mathbb{R}^{2}$. We state one of the conditions required in [11], which does not hold in this case: "there exist $\mu>0$ and $\gamma>0$ such that for all $x \in(\Sigma+\mu B) \cap K^{c}$ and $\zeta \in x-\pi_{\Sigma}(x)$ there exists $v \in F(x)$ such that $\langle v, \zeta\rangle \leq-\gamma\|\zeta\|$ ". Assume by contradiction that there exist $\mu>0$ and $\gamma>0$ such that for $(0,-a) \in \mu B \cap K^{c}(a>0)$ we can find $\left(v_{1}, v_{2}\right) \in F(x, y)$ with $\left\langle\left(v_{1}, v_{2}\right),(0,-a)\right\rangle \leq-\gamma a$. Hence, $v_{2} \geq \gamma>0$, which contradicts the fact that $\left(v_{1}, v_{2}\right) \in F(x)$.

However, our hypotheses from Theorem 3.2 hold. Indeed, for $(x, y) \in K$, $(x, y) \neq(0,0)$, let us take $u=-\frac{(x, y)}{\|(x, y)\|}$, which obviously belongs to $F(x, y)$ and satisfies $\langle(x, y), u\rangle=-\|(x, y)\|$. Also, there exist $h_{n}=\frac{1}{n} \downarrow 0$ and $\left(p_{n}, q_{n}\right)=\left(0, \frac{1}{n}\right) \rightarrow(0,0)$ such that $\left(x-x h_{n}+h_{n} p_{n}, y-h_{n} y+h_{n} q_{n}\right) \in K$, for each $n \in \mathbb{N}^{*}$, that is $-(x, y) \in \mathcal{T}_{K}(x, y)$, so $u \in \mathcal{T}_{K}(x, y)$.

Example 4.2. Consider $K=\left\{(x, y) ; y \geq-x^{2}\right\}, \Sigma=\{(0,0)\}$ and $F(x, y)=B$ for all $(x, y) \in \mathbb{R}^{2}$. It easy to see that $\Sigma$ is not admissible for $K$, so, the results from [11] can not be applied. We recall that $\Sigma$ is an admissible set for $K$ iff there exists $\rho>0$ such that for all $\alpha \in \Sigma \cap$ bdryS we have $N_{\Sigma, K}^{P, \rho}(\alpha) \subset \mathcal{T}_{K}(\alpha)$, where

$$
N_{\Sigma, K}^{P, \rho}(\alpha)=\left\{t(x-\alpha) ; x \in K \cap B(\alpha ; \rho), \alpha \in \pi_{\Sigma}(x), t \geq 0\right\} .
$$

However, the conditions required in Theorem 3.2 are satisfied. Indeed, for $(x, y) \in K,(x, y) \neq(0,0)$, take $u=\frac{1}{\|(x, y)\|}\left(-\frac{1}{4} x,-y\right)$. Obviously, $\|u\| \leq$ 1. Moreover, there exist $h_{n}=\frac{1}{n},\left(p_{n}, q_{n}\right)=\left(0, \frac{1}{n}\right), n \in \mathbb{N}^{*}$ such that $(x, y)+h_{n}\left(-\frac{1}{4} x,-y\right)+h_{n}\left(p_{n}, q_{n}\right) \in K$ for all $n \in \mathbb{N}^{*}$, i.e. $\left(-\frac{1}{4} x,-y\right) \in$ $\mathcal{T}_{K}(x, y)$, hence $u \in \mathcal{T}_{K}(x, y)$. Also, we have that $\langle(x, y), u\rangle \leq-\frac{1}{4}\|(x, y)\|$, so inequality (3.4) is satisfied for $\gamma=\frac{1}{4}$.

Acknowledgements. The author was supported by CNCSIS grant PD_ 289/2010.

\section{REFERENCES}

1. Aubin, J.-P.; Frankowska, H. - Set-Valued Analysis, Systems \& Control: Foundations \& Applications, 2, Birkhäuser Boston, Inc., Boston, MA, 1990. 
2. BARdi, M.; FAlCONE, M. - An approximation scheme for the minimum time function, SIAM J. Control Optim., 28 (1990), 950-965.

3. BRÉzIS, H.; BRowder, F.E. - A general principle on ordered sets in nonlinear functional analysis, Advances in Math., 21 (1976), 355-364.

4. CAnnarsa, P.; CÂRJĂ, O. - On the Bellman equation for the minimum time problem in infinite dimensions, SIAM J. Control Optim., 43 (2004), 532-548.

5. CÂRJĂ, O. - The minimal time function in infinite dimensions, SIAM J. Control Optim., 31 (1993), 1103-1114.

6. CÂRJ $\breve{A}$, O. - On the minimum time function and the minimum energy problem; a nonlinear case, Systems Control Lett., 55 (2006), 543-548.

7. CÂRJĂ, O.; LAZU, A. - Regularity of the minimal time function for the heat equation, An. Ştiinţ. Univ. "Al.I. Cuza" Iaşi, Mat. (N.S.), 55 (2009), 355-364.

8. CÂrJă, O.; Necula, M.; Vrabie, I.I. - Viability, Invariance and Applications, North-Holland Mathematics Studies, 207, Elsevier Science B.V., Amsterdam, 2007

9. CÂRJĂ, O.; URSESCU, C. - The characteristics method for a first order partial differential equation, An. Ştiinţ. Univ. "Al.I. Cuza" Iaşi, Secţ. I a Mat., 39 (1993), 367-396.

10. Clarke, F.H.; Ledyaev, Yu.S.; Stern, R.J.; Wolenski, P.R. - Nonsmooth Analysis and Control Theory, Graduate Texts in Mathematics, 178, Springer-Verlag, New York, 1998.

11. Nour, C.; Stern, R.J. - Regularity of the state constrained minimal time function, Nonlinear Anal., 66 (2007), 62-72.

12. Petrov, N.N. - On the Bellman function for the time-optimal process problem, J. Appl. Math. Mech., 34 (1970), 785-791.

13. SoraviA, P. - Pursuit-evasion problems and viscosity solutions of Isaacs equations, SIAM J. Control Optim., 31 (1993), 604-623.

14. Veliov, V.M. - Lipschitz continuity of the value function in optimal control, J. Optim. Theory Appl., 94 (1997), 335-363.

15. Wolenski, P.; Zhuang, Y. - Proximal analysis and the minimal time function, SIAM J. Control Optim., 36 (1998), 1048-1072. 